\title{
Review of: "Data Valence"
}

David Graham ${ }^{1}$

1 University College Dublin

Potential competing interests: The author(s) declared that no potential competing interests exist.

An interesting definition to "Data Valence" that could, perhaps, be analogous to/transposed to "Data Gravity," that is, the innate nature of data to attract value via applications (material, digital, services).

Given the nature of time and advancement since this original definition was published, perhaps we can update this with more contemporaneous meaning. 